\title{
Reflets
}

Revue ontaroise d'intervention sociale et communautaire

\section{Économie sociale et solidaire}

\section{Lucie Bélanger et Danielle Fournier}

Volume 3, numéro 2, automne 1997

Visibles et Partenaires : Pratiques et recherches féministes

URI : https://id.erudit.org/iderudit/026177ar

DOI : https://doi.org/10.7202/026177ar

Aller au sommaire du numéro

Éditeur(s)

Reflets : Revue ontaroise d'intervention sociale et communautaire

ISSN

1203-4576 (imprimé)

1712-8498 (numérique)

Découvrir la revue

Citer cet article

Bélanger, L. \& Fournier, D. (1997). Économie sociale et solidaire. Reflets, 3(2),

144-155. https://doi.org/10.7202/026177ar

Tous droits réservés (C) Reflets : Revue ontaroise d'intervention sociale et communautaire, 1997

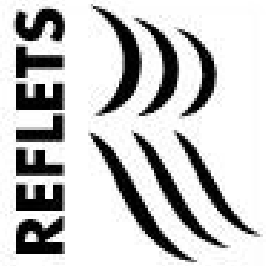

Ce document est protégé par la loi sur le droit d'auteur. L’utilisation des services d'Érudit (y compris la reproduction) est assujettie à sa politique d'utilisation que vous pouvez consulter en ligne.

https://apropos.erudit.org/fr/usagers/politique-dutilisation/ 


\section{Économie sociale et solidaire}

Lucie Bélanger, coordonnatrice à la recherche, Relais-femmes

Danielle Fournier, École de service social, Université de Montréal

\section{Économie sociale et solidaire, un projet féministe?}

"La marche a été un véritable raz de marée de solidarité.»
Le but de cet article, dans le cadre du colloque Visibles et Partenaires, est d'entrevoir en quoi l'économie sociale et solidaire fait appel aux femmes et s'appuie de façon particulière sur leurs pratiques. Or, nous ne pouvons pas parler des liens entre l'économie sociale et solidaire et le mouvement féministe sans effectuer un retour sur la marche des femmes contre l'appauvrissement qui s'est déroulée au Québec en juin 1995 : la marche Du Pain et des roses. Cet événement s'inscrit dans le combat historique des femmes pour changer l'ordre des choses. En ce sens, la marche fait écho au slogan lancé lors de la journée du 8 mars, à Québec, au début des années 1980: Bien sûr qu'on est folle, on veut changer le monde. Ce cri unanime, ce slogan, reste toujours profondément inscrit dans les grandes comme dans les petites actions des femmes. Oui, nous, les femmes, portons toujours ce défi de changer le monde.

La marche a été un véritable raz de marée de solidarité. Ce projet a touché le cœur des hommes et des femmes, des jeunes et des moins jeunes qui se sont rassemblés pour marcher: un geste simple pouvant être accompli par plusieurs. Toutes ne pouvaient pas marcher pendant les dix jours que durait la marche, mais tous et toutes pouvaient, à un moment ou à un autre, être sur le bord de la route pour encourager les marcheuses, leur préparer des repas, avoir une chambre pour les accueillir. C'était un événement où chacun et chacune pouvait trouver sa place et se joindre à la 
"...la marche est une manifestation visant à obtenir des réponses et des engagements de nos gouvernants..." marche pour construire une société différente. L'économie sociale et solidaire n'est pas née à ce moment, mais la marche a été le creuset qui l'a dévoilée au grand jour ${ }^{1}$.

Il est important de se rappeler que la marche est une manifestation visant à obtenir des réponses et des engagements de nos gouvernants par rapport à neuf revendications:

- la création d'un vaste programme d'infrastructures sociales;

- l'augmentation du salaire minimum à 8,30 \$ l'heure;

- l'application de la Loi des normes du travail pour toutes les personnes soumises à des mesures d'employabilité ou à l'aide sociale;

- une loi pour l'équité salariale;

- la réduction du parrainage pour les femmes immigrantes;

- un système de perception automatique des pensions alimentaires;

- la création d'unités de logement social, soit un minimum de 1500 unités par année;

- l'accès aux services et programmes de formation générale et professionnelle pour toutes les personnes y compris les «sanschèques $^{2} »$;

- le gel des frais de scolarité et l'augmentation des bourses d'étude.

Ces revendications, définies collectivement, sont le fruit d'un long processus de discussions. Ces revendications, suggérant un «virage libérateur», devaient être concrètes, gagnables à court terme, afin de continuer à changer le monde. Les propos de Lorraine Guay, une marcheuse et une inspiratrice, sont particulièrement éclairants à cet égard.

En ce sens, la marche des femmes participait au projet porté par de multiples mouvements sociaux, féministes, communautaires, autonomes, syndicaux, écologiques, de solidarité internationale, qui travaillent quotidiennement, et j'insiste sur le quotidiennement, à bâtir une société où le sens de la vie individuelle et collective l'emporte sur la logique productiviste: cette logique écologiquement solidaire, guerrière, sexiste, à laquelle la condamne le néolibéralisme triomphant. Une société exempte de sexisme, de racisme, et où la solidarité, la démocratie et l'égalité entre hommes et femmes constituent à la fois des fins et des moyens. On marchait, on a marché et on continue de travailler pour une société qui ne 
«En effet, chacune des revendications faites à cette occasion confronte, sous un angle précis, le capitalisme et le patriarcat engagés au coude à coude dans la grande cabale de la lutte au déficit." saurait se renouveler sans le dynamisme des mouvements sociaux, à la fois critiques et porteurs d'alternatives. Une société qui réaffirme le rôle incontournable et indispensable de l'État pour assurer à tous et à toutes une citoyenneté de faits et de droits; une société où il ne saurait y avoir de développement politique, social, culturel et économique sans la participation pleine et entière des femmes ${ }^{3}$.

En effet, chacune des revendications faites à cette occasion confronte, sous un angle précis, le capitalisme et le patriarcat engagés au coude à coude dans la grande cabale de la lutte au déficit. Chacune est l'élément d'un projet de riposte global et cohérent au néo-libéralisme. Il s'agit de neuf revendications formant un tout mutuellement inclusif. En effet, on ne peut pas penser aux programmes d'infrastructures sociales sans songer à l'équité salariale, sans augmenter le salaire minimum, sans évoquer un statut d'autonomie réelle pour les immigrantes, et ainsi de suite. Or, si nous oublions l'ensemble des revendications comme projet d'économie sociale et solidaire, comme forme de résistance au néo-libéralisme, et que nous les prenons une à une, de façon isolée, nous nous trouvons à en édulcorer le sens.

Ce découpage en morceaux trahit l'attitude sous-jacente aux actions gouvernementales par rapport au mouvement d'économie sociale et solidaire ${ }^{4}$. Une telle attitude des gouvernements a suscité d'âpres débats au sein du mouvement. Certaines y ont vu une oeuvre de récupération par le pouvoir, alors que d'autres persistaient à dire que le projet constitue une alternative réelle à l'appauvrissement; certaines ont évoqué la naïveté politique du mouvement, alors que d'autres ont défendu sa vision critique. À celles-ci comme à celles-là, il est bon de rappeler que le projet forme un ensemble indissociable.

Cela étant dit, nous voudrions nous pencher sur une de ces revendications, soit le programme d'infrastructures sociales. Cette revendication vient d'un questionnement suscité, en quelque sorte, par le programme d'infrastructures routières et autres, mis de l'avant par les libéraux de Jean Chrétien lors de la campagne électorale fédérale de 1993, qui planifiait d'injecter dans l'économie plus de 25 millions de dollars par année pour une période de cinq ans. Le mouvement national des garderies fut le premier à établir le lien 
entre le programmes d'infrastructures de Jean Chrétien et les infrastructures sociales. Repris par une large coalition engagée sur le terrain du social, ce lien ou ce «jeu de mots» cherchait à rappeler aux libéraux, alors assurés de la victoire électorale, que cette coalition d'organismes participait, elle aussi, à la construction d'infrastructures visant à garantir la qualité du tissu social de nos communautés. Et ce sont les femmes qui y sont mobilisées en très grande majorité.

\section{Infrastructure sociale et économie solidaire}

"...ce n'est pas parce que le terme "économie sociale et solidaire» est aujourd'hui sur toutes les lèvres, qu'il vient de naître...»
Cette revendication d'un programme d'infrastructure sociale, que l'on associe volontiers aujourd'hui à l'économie sociale et solidaire, trouve ses racines dans la construction des mouvements communautaire, populaire et féministe, qui sont engagés quotidiennement et historiquement dans la lutte contre l'appauvrissement, pour la défense des droits sociaux, pour la promotion de l'autonomie, pour l'égalité et les droits des femmes et pour la recherche et la mise en œuvre de solutions alternatives dans le champ social, politique et économique.

Par conséquent, ce n'est pas parce que le terme «économie sociale et solidaire» est aujourd'hui sur toutes les lèvres, qu'il vient de naitre, comme une chose venue de nulle part. Cette revendication d'infrastructures sociales est le résultat d'une longue pratique d'organismes communautaires, féministes et syndicaux. Celle-ci cherche à redonner à la personne la centralité dans les choix économiques, politiques et sociaux.

S'il importe d'expliquer comment s'élabore concrètement la revendication d'infrastructures sociales, maintenant articulées au projet d'économie sociale et solidaire, il est tout aussi important de ne pas oublier que ce n'est pas une revendication isolée ou sectorielle. Nous insistons sur ce point. S'inscrivant dans un projet, cette revendication est la remise en cause radicale des grands paradigmes économiques: le dieu Marché, la gouvernance 
"L'économie sociale et solidaire veut placer la ou les personnes, particulièrement les personnes marquées par l'exclusion, au centre des projets économiques, sociaux et politiques, en tant qu'acteures et acteurs de leur histoire." stratégique, la lutte au déficit, la compétitivité, etc. Ici, les mots ont tout leur poids. Nous parlons bien d'économie sociale et solidaire, et non d'une quelconque adaptation "humaniste» néolibérale, voire d'une soupape de sûreté à la crise du fordisme des années 1980. L'économie sociale et solidaire veut placer la ou les personnes, particulièrement les personnes marquées par l'exclusion, au centre des projets économiques, sociaux et politiques, en tant qu'acteures et acteurs de leur histoire.

Depuis plus de cinq ans, les centres de femmes adoptent une démarche visant à sensibiliser les femmes au fait qu'elles sont des agentes dans l'économie, des agentes de développement de la société, des agentes qui interviennent politiquement. Les femmes ne sont pas seulement des «sociales», celles qui rapiècent ce que d'autres décousent ou déchirent; elles sont à construire un projet de société à travers ce qu'elles initient quotidiennement. C'est ce qu'évoque une féministe égyptienne en parlant des femmes qui travaillaient avec elle dans un bidonville du Caire et en les décrivant comme des artisanes de la solidarité dans leur communauté. Effectivement, notre tâche historique a été, et demeure pour plusieurs d'entre nous, de prendre soin (to care). Nous avons développé dans ce champ des expertises particulières et précieuses qui doivent devenir des outils permettant aux femmes d'accéder à l'autonomie financière et politique.

Aussi, lorsqu'on parle d'une société qui renait au cœur des projets d'infrastructures sociales, c'est toute la qualité du tissu social, tout le retissage de la solidarité qui est en cause. Nous ne parlons pas d'un secteur marginal qui offre une bouffée d'oxygène à un système économico-politique qui éprouve des difficultés à respirer, nous parlons d'un projet de société qui a pour centre et vecteur d'animation, les personnes d'abord.

Par la revendication d'un programme d'infrastructures sociales, nous revendiquons de vrais emplois, car il s'agit d'un travail réel et nécessaire. Ce programme doit donc être un espace créateur d'emplois qualifiés et qualifiants, qui répondent aux besoins de santé, d'éducation, de culture, de transports collectifs, de logements sociaux... Ces foyers de besoins fondamentaux sont autant de foyers de création d'emplois orientés vers la qualité de vie des communautés. 


\section{Économie sociale et solidaire et justice sociale}

"...qu'une économie qui ne saurait pas se mettre au rythme de la construction de la justice sociale n'a aucun sens...»
L'économie sociale et solidaire, c'est aussi justice sociale et économie, alors même que les "grands» spécialistes de l'économie cherchent à nous convaincre que l'un, c'est philosopher, et l'autre, c'est s'occuper de choses sérieuses. Or, il importe de rappeler qu'une économie qui ne saurait pas se mettre au rythme de la construction de la justice sociale n'a aucun sens, qu'elle oublie ce qui est fondamental et vital. Comme société, il faut passer de l'obsession du productivisme à l'obsession quotidienne de la justice, de la solidarité et de la dignité des personnes. Nous ne sommes pas économistes, mais on ne nous persuadera pas que l'économie ne peut pas se concevoir selon des paramètres de justice sociale.

S'étant vues confier le soin quotidien des personnes, les femmes en sont devenues les expertes; et nous devons, pour exercer cette responsabilité, maitriser les leviers politiques permettant la mise en œuvre, au quotidien, d'un modèle de développement qui priorise d'abord le partage $\mathrm{Du}$ Pain et des roses avec les exclus: femmes, hommes et enfants. La maitrise de ces leviers vise à assurer que le partage de la richesse «accaparée» redevienne un partage $D u$ Pain et des roses pour toutes et tous.

La mobilisation des femmes n'a pas lieu seulement au Québec, mais en Ontario, au Canada, au Nord comme au Sud, car ce sont les femmes qui vivent le plus crûment, au quotidien, les impacts dévastateurs du néo-libéralisme. En effet, ce sont les avancées réalisées par les femmes au cours des dernières décennies, y compris celles obtenues lors de la conférence mondiale de Beijing, qui sont menacées au profit de la lutte au déficit. Certes, on admet volontiers que la production non salariée des femmes est excessivement importante, mais on ne la reconnait pas pour en faire un pivot de l'accès des femmes à l'autonomie politique et économique, notamment par la création d'emplois véritables qui tiennent compte des expertises, des expériences, des compétences, des connaissances acquises par les femmes dans le champ de cette production. 
«En somme, on glorifie le maternage privé et social dans le cadre de «jobinettes»...»
Lorsque le pouvoir souscrit à toutes ces qualifications, c'est pour chercher à déconstruire nos acquis sociaux et nos politiques sociales. C'est aussi pour exiger des femmes qu'elles accomplissent ce travail à rabais, comme aidantes naturelles, dans un contexte d'isolement des familles ou d'activités bénévoles. En somme, on glorifie le maternage privé et social dans le cadre de «jobinettes», et non pas celui d'emplois véritables reconnus, comme ceux existant dans le secteur public ou les organismes communautaires. Cela rend plus actuelle la thèse du ressac (backlash). À tout le moins, peut-on y détecter l'œuvre du patriarcat qui cherche à développer des formes «modernes» d'appropriation de notre production domestique, en réponse à son désengagement social. Aussi, nous disons non: "Ça suffit la gestion de la misère. Nous exigeons Du Pain et des roses».

Nous, les femmes, comprenons l'économie. Nous la comprenons à partir d'impératifs quotidiens: nourrir, prendre soin, vivre. Ce terreau doit être le lieu de naissance de modèles économiques porteurs de plus de justice, d'égalité, de dignité. Comment relever ce défi au cœur de l'action?

\section{Économie sociale et solidaire: principes et pratiques}

L'économie sociale et solidaire ou la revendication des femmes concernant un programme d'infrastructures sociales est l'objet de tensions et de tiraillements au sein du mouvement des femmes, voire même du mouvement communautaire. En effet, ce projet de société ne recueille pas l'adhésion aveugle de toutes les femmes. Les femmes sont conscientes des dangers qui guettent un tel projet, en raison de la conjoncture économique actuelle (palliatif à la crise des finances publiques ou sorte de passerelle pour l'emploi par des mesures d'employabilité sous-payées, disqualifiées). C'est pourquoi ce projet fait l'objet d'une intense discussion pour cerner l'orientation de l'économie sociale et solidaire. Cette discussion est une condition essentielle de sa vitalité dans le cadre d'un processus démocratique. 
"L'économie sociale et solidaire vise le développement d'un espace économique différent...»
L'économie sociale et solidaire vise le développement d'un espace économique différent; de ce fait, elle s'efforce de produire autrement, tant dans la manière de produire que dans le type de produits ou de services offerts. L'économie sociale et solidaire cherche à mettre en place certains biens et services d'utilité collective, qui soient multiformes et qui reposent sur une valorisation du savoir des communautés, du savoir des femmes, puisque ce sont elles qui, en priorité, vont définir, construire et produire.

L'économie sociale et solidaire est un secteur économique qui a son propre système de production, sa propre logique. Différente du secteur marchand et du secteur public, l'économie sociale et solidaire interpelle, dérange et dérangera l'économie marchande et le secteur public,ce qui ne l'empêche pas de nourrir des liens constants ou de développer des partenariats avec l'une ou l'autre de ces sphères d'activités. Par exemple, certains projets sur le terrain de l'économie sociale et solidaire, liés davantage à des domaines tels la foresterie et l'environnement, sont en interface avec l'économie marchande 5 . Par ailleurs, d'autres activités, entreprises ou ressources développent une interface avec la production de type public, notamment dans les domaines de la santé et des services sociaux. Nous songeons ici au domaine de l'entretien ménager à domicile ${ }^{6}$. Donc, l'économie sociale et solidaire est multiforme et tenter de l'emprisonner dans un modèle ou une pratique unique serait signer son arrêt de mort.

Malgré ces balises, qui permettant de mieux situer le contexte, nous devons toutefois poursuivre notre effort de définition et préciser la «nature», la logique propre de l'économie sociale et solidaire en tant que système de production, afin qu'elle ait sa place au soleil au même titre que les économies marchande et publique. Cette tâche de définition s'impose d'autant plus que si nous ne l'accomplissons pas, d'autres acteurs dans la société s'en chargeront et façonneront l'économie sociale et solidaire à leur image. Il ne faut pas oublier que nous sommes plongés dans un contexte de crise : crise du travail, crise des finances publiques et obsession du déficit zéro. Il y a donc danger que des dérives ou des détournements de sens se produisent. 
"...l'économie sociale et solidaire doit répondre à des besoins sociaux, conviviaux, relationnels et d'empowerment.»
Nous pourrions définir l'économie sociale et solidaire comme des activités, des entreprises, des organismes issus de l'entreprenariat collectif qui doivent satisfaire aux principes suivants:

- avoir comme finalité,le service des membres et des collectivités, plutôt que de simplement viser les profits ou la rentabilité financière;

- avoir une gestion collective et solidaire, et donc différente de la manière dont on produit dans les services publics (par exemple, gestion par clientèle ou groupe-cible);

- respecter un processus de prise de décision démocratique;

- accorder la primauté aux personnes et au travail sur le capital dans la répartition des surplus et des revenus; et

- élaborer des activités qui reposent sur des principes de participation, de prise en charge, de responsabilité individuelle et collective.

Ainsi définie, l'économie sociale et solidaire doit répondre à des besoins sociaux, conviviaux, relationnels et d'«empowerment». C'est pourquoi l'économie sociale et solidaire est une forme de contestation du pouvoir marchand et du pouvoir public. Elle représente le choix des communautés qui se prennent en main pour échapper aux logiques marchandes et publiques. Le développement de l'économie sociale et solidaire cherche à combler certaines lacunes, notamment un certain déficit démocratique, un sous-développement social, culturel, économique, politique et environnemental dans plusieurs communautés et collectivités. Cela dit, la mise en place de l'économie sociale et solidaire comporte ses exigences.

Le principal défi, c'est la création d'emplois véritables d'utilité sociale, qui permettent de rendre visible et de reconnaître à sa juste valeur le travail assumé par les femmes. Pour ce faire, nous devons requalifier ce qui a été déqualifié. La discrimination systémique est toujours à l'oeuvre, tout comme le sexisme est toujours présent.

Depuis dix ans, l'État et ses services publics délaissent plusieurs domaines d'intervention sociale:le sida, l'itinérance, etc. Or, dans les faits, que se passe-t-il le plus souvent? Au mieux, c'est l'économie sociale et solidaire qui investit ce champ et s'en charge. 
Au pire, c'est le travail au noir. Devant ce dilemme, que fait-on? On peut continuer à faire du travail au noir et ainsi échapper à la visibilité et à la comptabilité nationale de l'État ou du marché. Par ailleurs, on peut travailler à rendre visible, à créer des emplois de qualité qui témoignent de l'immense valeur et de la richesse économique de cette production sociale. C'est la position qu'adopte l'économie sociale et solidaire.

De là découle un autre enjeu, soit la nécessité de développer des indicateurs sociaux qui sont propres à l'économie sociale et solidaire et qui peuvent aussi se traduire en langage comptable. Il faut questionner l'exigence et l'obligation du rendement financier pour une partie importante de l'économie sociale et solidaire, car le rendement financier est issu d'une logique marchande

«Il va falloir aussi faire preuve d'imagination et développer ce que nous appelons un financement hybride pour les projets d'économie sociale et solidaire..." spéculative. Il va falloir aussi faire preuve d'imagination et développer ce que nous appelons un financement hybride pour les projets d'économie sociale et solidaire, c'est-à-dire un financement qui provient de plusieurs sources. Bien sûr, l'État sera mis à contribution, mais nous croyons que le marché doit, lui aussi, investir dans l'économie sociale et solidaire. Cela tient de sa responsabilité sociale mutuelle. Ainsi, le capital nécessaire au financement des projets viendrait à la fois de l'État, du marché et de la communauté. Il y a plusieurs projets qui, malgré l'activité économique qu'ils génèrent, ne pourront jamais être rentables au sens financier du terme, mais qui sont et seront socialement rentables.

De plus, l'économie sociale et solidaire assume, selon nous, un élément essentiel dans notre société actuelle: elle permet de refaire le tissu social par de nouveaux efforts de solidarité. Ceci nous apparaît primordial. En effet, l'économie sociale et solidaire joue un rôle essentiel dans toutes les questions touchant la citoyenneté, dans la mesure où c'est par le travail, rendu visible, que s'accomplit l'intégration des individus.

Certes, les dangers demeurent. C'est pourquoi la discussion se poursuivra au sein des groupes de femmes, tout en menant l'expérience de l'économie sociale et solidaire, car il ne s'agit pas seulement de discuter, mais également d'expérimenter. Il faut dire que l'économie sociale et solidaire n'est pas la panacée à tout ce que nous vivons. Il faut se rendre compte qu'il s'agit là d'une 
"...il faut redéfinir un véritable contrat social...» alternative parmi d'autres et qu'il est nécessaire de mener nos luttes sur plusieurs fronts. Il importe de poursuivre notre questionnement sur le secteur marchand, de questionner les règles du jeu à l'heure actuelle, de trouver des solutions différentes de celles proposées par le courant néo-libéral face à la crise du travail, de réfléchir sur le rôle et la place de l'État. En somme, il faut redéfinir un véritable contrat social à l'intérieur duquel l'économie sociale et solidaire aura un rôle important à jouer dans toute la question de la redistribution et du partage de la richesse collective. Du Pain et des roses pour tous et toutes!

\section{Bibliographie}

AUBRU, F. et J. CHARREST (1995). Développer l'économie solidaire. Éléments d'orientation, Montréal, Confédération des syndicats nationaux.

BELLEAU, J. (1995). Économie sociale: quelques éléments de réflexion ou une autre version de "Môman à travaille pas, à trop d'ouvrage», Montréal, l'R des Centres de femmes du Québec.

COLLECTIF CLIO (1982). L'histoire des femmes au Québec depuis quatre siècles, Montréal, Quinze.

DEMUSTIER, Danièle (1995). "L'économie sociale toujours à réinventer», Paris, Alternatives économiques.

GROUPE DE LISBONNE (1995). Limites à la compétitivité, Montréal, Boréal.

GUAY, Lorraine (1996). «Le mouvement communautaire «entre l'espoir et le doute» face à l'économie sociale», texte de l'exposé donné au colloque organisé par l'Association d'économie politique qui a eu lieu les 18 et 19 octobre 1996, sur le thème Au-delà du néo-libéralisme, quel rôle pour les mouvements sociaux?

INTERACTION COMMUNAUTAIRE (1996). L'économie solidaire: dossier France-Québec, no 36-37.

LAVILLE, Jean-Louis (1992). «Les services de proximité en Europe», Syros - Alternatives, Paris.

LAVILLE, Jean-Louis (1994). «L'économie solidaire: une perspective internationale», Paris, Desclée de Brouwer.

LAVILLE, Jean-Louis (1996). «L'économie solidaire», Paris, Desclée de Brouwer.

PERRET, B. (1994). «Politiques publiques et mobilisation collective», dans B. Eme et J.L. Laville Cohésion sociale et emploi, Paris, Desclée de Bouwer.

ROUSTANG, G., J.L. LAVILLE, B. EME, D. MOTHE et B. PERRET (1996). Vers un nouveau contrat social, Paris, Desclée de Brouwer.

VANDELAC, L., D. BÉLISLE, A. GAUTHIER et Y. PINARD (1984). Du travail et de l'amour: les dessous de la production domestique, Montréal, Éd. Coopératives Saint-Martin.

VIENNEY, C. (1994). L'économie sociale, Paris, La Découverte.

WARING, M. (1995). Who's Counting? Marylin Waring on Sex, Lies \& Global Economics (enregistrement vidéo), Montréal, National Film Board. 


\section{Notes}

1. NDLR: On se souviendra que c'est à la suite de cette marche que le gouvernement Bouchard a inscrit un chantier portant sur l'économie sociale, qui a été dirigé par Nancy Neantam, au programme d'une série de conférences regroupant les principaux intervenants sociaux et du monde des affaires au Québec.

2. NDLR: Les «personnes avec chèques» sont des personnes recevant des prestations d'assurance-emploi ou d'aide sociale qui ont accès à des services de formation. Les «personnes sans-chèques» sont les personnes démunies, pauvres, sans-abri, etc. En somme, ce sont les personnes exclues du secteur central du marché du travail, secteur qui comporte de bons salaires, des avantages sociaux, la sécurité d'emploi, etc. Ce sont aussi des femmes au foyer.

3. Cette citation est tirée du document intitulé Un rappel, un appel, rédigé par Lorraine Guay en 1997. Ce document n'a pas été publié.

4. NDLR: Peu de temps après la tenue de la Marche, le gouvernement québécois a accédé à l'une des revendications des femmes, soit la mise en place d'un système de perception automatique des pensions alimentaires.

5. NDLR: On songe ici à La Maison verte de Hearst, à la coopérative Convergence ou à la coopérative de travail marrainée par le Collectif des femmes francophones du NordEst ontarien. Ce sont toutes des coopératives mises sur pied par un groupe de femmes.

6. NDLR: Par exemple, on peut songer aux services d'aide familiale que cherche à établir le gouvernement ontarien dans le cadre de sa politique sur les soins de longue durée. Pour plus de détails, on pourra se référer au document Exigences provinciales concernant le processus de demande de proposition pour la prestation de services, de fournitures et d'équipement à domicile, du ministère de la Santé de l'Ontario, division des soins de longue durée, mai 1996. 